\title{
2MASS J06164006-6407194: THE FIRST OUTER HALO L SUBDWARF
}

\author{
Michael C. Cushing ${ }^{1}$, Dagny Looper ${ }^{1}$, Adam J. Burgasser ${ }^{2}$, J. Davy KirkPatrick ${ }^{3}$, Jacqueline Faherty ${ }^{4}$, \\ Kelle L. Cruz ${ }^{5}$, Anne Sweet ${ }^{5}$, and Robyn E. Sanderson ${ }^{2}$ \\ ${ }^{1}$ Institute for Astronomy, University of Hawai'i, 2680 Woodlawn Drive, Honolulu, HI 96822, USA; mcushing@ ifa.hawaii.edu \\ ${ }^{2}$ Massachusetts Institute of Technology, Kavli Institute for Astrophysics and Space Research, Building 37, Room 664B, 77 Massachusetts Avenue, Cambridge, MA \\ 02139, USA \\ ${ }^{3}$ Infrared Processing and Analysis Center, M/S 100-22, California Institute of Technology, Pasadena, CA 91225, USA \\ ${ }^{4}$ Department of Physics and Astronomy, Stony Brook University, Stony Brook, NY, 11794, USA \\ ${ }^{5}$ Department of Astronomy, Caltech, MC 105-24, Pasadena, CA 91125, USA \\ Received 2009 January 5; accepted 2009 February 5; published 2009 April 20
}

\begin{abstract}
We present the serendipitous discovery of an L subdwarf in the Two Micron All Sky Survey (2MASS) J06164006-6407194, in a search of the 2MASS for T dwarfs. Its spectrum exhibits features indicative of both a cool and metal poor atmosphere including a heavily pressure-broadened $\mathrm{K}$ I resonant doublet, Cs I and Rb I lines, molecular bands of $\mathrm{CaH}, \mathrm{TiO}, \mathrm{CrH}, \mathrm{FeH}$, and $\mathrm{H}_{2} \mathrm{O}$, and enhanced collision induced absorption of $\mathrm{H}_{2}$. We assign 2MASS J0616-6407 a spectral type of sdL5 based on a comparison of its red optical spectrum to that of near solarmetallicity L dwarfs. Its high proper motion $\left(\mu=1^{\prime \prime} .405 \pm 0.008 \mathrm{yr}^{-1}\right)$, large radial velocity $\left(V_{\mathrm{rad}}=454 \pm 15 \mathrm{~km} \mathrm{~s}^{-1}\right)$, estimated $u, v, w$ velocities $(94,-573,125) \mathrm{km} \mathrm{s}^{-1}$ and Galactic orbit with an apogalacticon at $\sim 29 \mathrm{kpc}$ are indicative of membership in the outer halo making 2MASS J0616-6407 the first ultracool member of this population.
\end{abstract}

Key words: infrared: stars - stars: individual (2MASS J0164006-6407194) - stars: low-mass, brown dwarfs subdwarfs

Online-only material: color figures

\section{INTRODUCTION}

Ultracool subdwarfs are metal-poor stars and brown dwarfs that have spectral types later than $\sim$ sdM7/esdM7 (Burgasser et al. 2005) and $T_{\text {eff }} \lesssim 3000 \mathrm{~K}$ (Leggett et al. 2000). Since cool subdwarfs are typically members of the thick disk or halo population, they exhibit large space motions relative to the Sun $\left(\langle V\rangle=-202 \mathrm{~km} \mathrm{~s}^{-1}\right.$; Gizis 1997) and thus are often identified as high proper-motion stars (e.g., Giclas et al. 1971, 1978; Luyten 1979; Pokorny et al. 2004; Lépine \& Shara 2005). Due to their intrinsic faintness and paucity in the solar neighborhood, only $\sim 40$ ultracool subdwarfs are currently known (Burgasser et al. 2007; Lépine \& Scholz 2008). Such small numbers stand in stark contrast to the over one thousand near solar-metallicity ultracool dwarfs (spectral types later than M7 and $T_{\text {eff }} \lesssim 2400 \mathrm{~K}$ ) that have been discovered in the Two Micron All Sky Survey (2MASS; Skrutskie et al. 2006), the Sloan Digital Sky Survey (SDSS; York et al. 2000), and the Deep Near-Infrared Southern Sky Survey (Epchtein et al. 1997), many of which populate the L and T spectral classes ${ }^{6}$ (Kirkpatrick 2005).

Of the roughly 40 ultracool subdwarfs known, only three, 2MASS J0532346 + 8246465 (hereafter 2MASS J0532+8246; Burgasser et al. 2003), 2MASS J16262034 + 3925190 (hereafter 2MASS J1626+3925; Burgasser 2004), and SDSS J125637022452 (hereafter SDSS J1256-0224; Sivarani et al. 2009) are classified as L subdwarfs. ${ }^{7}$ The spectra of all three L subdwarfs exhibit $\mathrm{Cs}$ I and $\mathrm{Rb}$ I lines, pressure-broadened $\mathrm{K}$ I lines, $\mathrm{CaH}$, $\mathrm{FeH}, \mathrm{CrH}$ and $\mathrm{H}_{2} \mathrm{O}$ bands, and enhanced collision induced $\mathrm{H}_{2}$ absorption (CIA, e.g., Linsky 1969; Borysow et al. 1997), all

\footnotetext{
6 A compendium of known $\mathrm{L}$ and $\mathrm{T}$ dwarfs is given at http://DwarfArchives.org.

7 LSR 1610-0040 was originally classified as an L subdwarf by Lépine et al (2003) but observations by Cushing \& Vacca (2006), Reiners \& Basri (2006), and Dahn et al. (2008b) indicate that it is probably a peculiar M dwarf/subdwarf binary.
}

features indicative of very cool (Kirkpatrick et al. 1999; Leggett et al. 2001; Reid et al. 2001) and metal-depleted atmospheres (Bessell 1982; Saumon et al. 1994). In addition, they have large proper motions ( $\left(0.6\right.$ to $\left.2^{\prime \prime} .6 \mathrm{yr}^{-1}\right)$ and radial velocities $(-130$ to $-260 \mathrm{~km} \mathrm{~s}^{-1}$ ) consistent with halo membership. Burgasser et al. (2008) recently measured the parallax of 2MASS J0532+8246 and confirmed, based on its Galactic orbit, that it is indeed a member of the (inner) halo. Although no spectral classification scheme exists for L subdwarfs, Burgasser et al. (2007, 2009) have tentatively assigned spectral types of sdL3.5, sdL4, and sdL7 to SDSS J1256-0224, 2MASS J1626+3925, and 2MASS $\mathrm{J} 0532+8246$, respectively, based on a comparison with the red optical spectra of the near solar metallicity L dwarf standards (Kirkpatrick et al. 1999).

In this paper, we present the discovery of a fourth L subdwarf, 2MASS J06164006-6407194 (hereafter 2MASS J0616-6407) found in a search of the 2MASS database for T dwarfs. The discovery of 2MASS J0616-6407, along with follow-up nearinfrared imaging and spectroscopy, and red-optical spectroscopy is presented in Section 2. In Section 3, we discuss the spectral classification and kinematics of 2MASS J0616-6407.

\section{OBSERVATIONS}

\subsection{Source Identification}

2MASS J0616-6407 was identified as a T dwarf candidate by Looper et al. (2007) in the course of a search for T dwarfs in the 2MASS Database. It was selected based on its very blue near-infrared color $\left(J-K_{s}<0.02\right)$ and lack of any optical counterpart within $5^{\prime \prime}$ in the USNO-A2.0 catalog or the Digital Sky Survey (DSS) I and II images. A finder chart for 2MASS J0616-6407 is given in Figure 1 and pertinent properties are listed in Table 1. Below we describe follow-up near-infrared imaging and spectroscopy, and red-optical spectroscopy. 


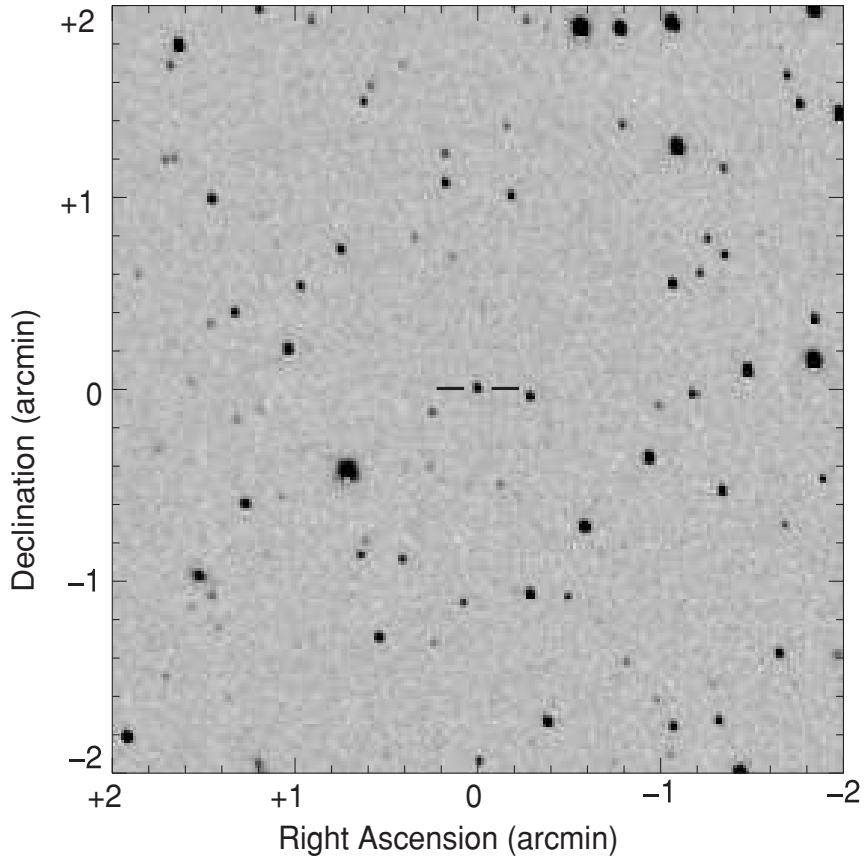

Figure 1. $J$-band image of the field centered around 2MASS J0616-6407 obtained with the CPAPIR on the $1.5 \mathrm{~m}$ CTIO on 2008 January 17 (UT). The field is $4^{\prime}$ on a side and north is up and east is to the left.

Table 1

Properties of 2MASS J0616-6407

\begin{tabular}{lc}
\hline \hline Parameter & Value \\
\hline \multicolumn{2}{c}{ Observed } \\
\hline$\alpha(\mathrm{J} 2000.0)^{\mathrm{a}}$ & $6^{h} 16^{m} 40^{\mathrm{s}} 06$ \\
$\delta(\mathrm{J} 2000.0)^{\mathrm{a}}$ & $-64^{\circ} 07^{\prime} 19^{\prime \prime} .4$ \\
$2 \mathrm{MASS} J$ & $16.403 \pm 0.113 \mathrm{mag}$ \\
$2 \mathrm{MASS} H$ & $16.275 \pm 0.228 \mathrm{mag}$ \\
$2 \mathrm{MASS} K_{s}$ & $>16.381^{\mathrm{b}} \mathrm{mag}^{-1}$ \\
$\mu_{\alpha}(\mathrm{J} 2000.0)$ & $1405 \pm 8 \mathrm{mas} \mathrm{yr}^{-1}$ \\
$\mu_{\delta}(\mathrm{J} 2000.0)$ & $-51 \pm 18 \mathrm{mas} \mathrm{yr}^{-1}$ \\
$V_{\text {rad }}$ & $454 \pm 15 \mathrm{~km} \mathrm{~s}^{-1}$ \\
\hline \multicolumn{2}{c}{} \\
\hline$d$ & Estimated \\
$V_{\text {tan }}$ & $57 \pm 9 \mathrm{pc}^{-1}$ \\
$u^{\mathrm{c}}$ & $379 \pm 60 \mathrm{~km} \mathrm{~s}^{-1}$ \\
$v$ & $94 \pm 10 \mathrm{~km} \mathrm{~s}^{-1}$ \\
$w$ & $-573 \pm 31 \mathrm{~km} \mathrm{~s}^{-1}$ \\
\hline
\end{tabular}

Notes.

a 2MASS coordinates at epoch 1998.95 (UT).

${ }^{\mathrm{b}}$ The read flag is 0 and the quality flag is $\mathrm{U}$ indicating that no flux was detected at the position and that the value is an upper limit.

c We follow the convention that the $u$ is positive toward the Galactic center $(l=0, b=0)$.

\subsection{Near-Infrared Spectroscopy}

2MASS J0616-6407 was observed with the Ohio State InfraRed Imager/Spectrometer (OSIRIS) mounted at the $4.1 \mathrm{~m}$ SOAR Telescope on 2007 March 12 (UT). We used the 1."0 wide slit which provides a resolving power of $R \equiv \lambda / \Delta \lambda \sim$ 1400 across the 1 to $2.2 \mu \mathrm{m}$ wavelength range in three spectral orders. A series of six $180 \mathrm{~s}$ integrations were obtained along the $24^{\prime \prime}$ long slit. The A0 V star HD 53191 was also observed to correct for telluric absorption and to flux-calibrate the final spectrum. The data were reduced using a modified version



Figure 2. Near-infrared spectra of 2MASS J1626+3925 (sdL4; Burgasser et al. 2007), 2MASS J0616-6407, and 2MASS J0532+8246 (sdL7; Burgasser et al. 2003). The spectra are normalized to unity at $1.3 \mu \mathrm{m}$ and offset by constants (dotted lines). Prominent atomic and molecular absorption features are indicated. The $\mathrm{S} / \mathrm{N}$ of the 2MASS J0616-6407 spectrum is not high enough to detect the $1.25 \mu \mathrm{m} \mathrm{K}$ I doublet.

(version 3.4) of the Spextool data reduction package (Cushing et al. 2004). Wavelength calibration was accomplished using the $\mathrm{OH}$ airglow lines in the science frames. The spectra from the three orders were then corrected for telluric absorption using the observations of the $\mathrm{A} 0 \mathrm{~V}$ standard stars and the technique described in Vacca et al. (2003). The three spectral orders were then merged into a single spectrum covering the $1.2-2.3 \mu \mathrm{m}$ wavelength range. The final spectrum was then rebinned to one pixel per resolution element in order to increase the signal-tonoise ratio $(\mathrm{S} / \mathrm{N})$ of the spectrum.

The spectrum of 2MASS J0616-6407 is shown in Figure 2 (middle). The $\mathrm{S} / \mathrm{N}$ of the spectrum is low, ranging from $\sim 8$ in the $J$ and $H$ bands to $\sim 4$ in the $K$ band, but is nevertheless adequate to confirm that 2MASS J0616-6407 is not a T dwarf (the $J-K_{s}$ color of 2MASS J0616-6407 corresponds to a spectral type of $\sim \mathrm{T} 4$ ) since the spectrum lacks the prominent $\mathrm{CH}_{4}$ bands found in the spectra of $\mathrm{T}$ dwarfs (Burgasser et al. 2002; Geballe et al. 2002). Also shown in Figure 2 are the spectra of the two other L subdwarfs with published spectra, 2MASS J0532+8246 (Burgasser et al. 2003) and 2MASS J1626+3925 (Burgasser 2004). All three spectra exhibit blue continua due to enhanced collision induced absorption (CIA) of $\mathrm{H}_{2}$ centered at $2.4 \mu \mathrm{m}$ and broad absorption bands of $\mathrm{H}_{2} \mathrm{O}$ centered at 1.4 and $1.9 \mu \mathrm{m}$ indicating that 2MASS J0616-6407 is an L subdwarf. 


\subsection{Red Optical Spectroscopy}

\subsubsection{Magellan/LDSS-3}

Red optical spectra of 2MASS J0616-6407 were obtained on 2006 May 10 (UT) with LDSS-3 on Magellan. The VPHred grism (660 lines $\mathrm{mm}^{-1}$ ) with a $0^{\prime} .75$ wide (4 pixels) longslit mask was used, with the slit aligned to the parallactic angle. This configuration provides $6050 \AA-10500 \AA$ spectra across the entire chip with an average resolving power of $R \approx 1800$ and dispersion along the chip of $\sim 1.2 \AA$ pixel $^{-1}$. The OG590 longpass filter was used to eliminate second-order light shortward of $6000 \AA$. Two slow-read exposures of $1800 \mathrm{~s}$ each were obtained at an airmass of 1.49-1.60. We also observed the G2 V star HD 44611 immediately after and at a roughly similar airmass (1.77) for telluric absorption correction. The flux standard LTT 7987 (aka GJ 2147; Hamuy et al. 1994) was observed on the previous night using an identical slit and grism combination. All spectral observations were accompanied by $\mathrm{HeNeAr}$ arc lamp and flat-field quartz lamp exposures for dispersion and pixel response calibration.

LDSS-3 data were reduced in the $\mathrm{IRAF}^{8}$ environment (Tody 1986). Raw images were first corrected for amplifier bias voltage, stitched together, and subtracted by a median-combined set of slow-read bias frames taken during the afternoon. These processed images were then divided by a median-combined, bias-subtracted and normalized set of flat field frames. The LTT 7987 and HD 44611 spectra were optimally extracted first using the APALL task with background subtraction. The spectrum of 2MASS J0616-6407 was then extracted using the $\mathrm{G}$ star dispersion trace as a template. Dispersion solutions were determined from arc lamp spectra extracted using the same dispersion trace; solutions were accurate to $\sim 0.08$ pixels, or $\sim 0.1 \AA$. Flux calibration was determined using the tasks STANDARD and SENSFUNC with observations of LTT 7987, adequate over the spectral range $6000 \AA-10000 \AA$. Corrections to telluric $\mathrm{O}_{2}(6855 \AA-6955 \AA \mathrm{B}$-band, $7580 \AA-7740 \AA \mathrm{A}$-band) and $\mathrm{H}_{2} \mathrm{O}(7160 \AA-7340 \AA, 8125 \AA-8350 \AA, 9270 \AA-9680 \AA)$ absorption bands were determined by linearly interpolating over these features in the $\mathrm{G}$ dwarf spectrum, dividing by the uncorrected spectrum, and multiplying the result with the spectrum of 2MASS J0616-6407. The two spectra of 2MASS J0616-6407 were then coadded to improve $S / N$. We measured the radial velocity of 2MASS J0616-6407 using the Rb I (7948 $)$, Na I (8183, $8195 \AA$ ), and Cs I (8521 ̊) lines. The observed line positions determined by Gaussian fits were compared against the rest wavelengths (Ralchenko et al. 2008) to derive a heliocentric radial velocity (after correcting for the Earth's orbital velocity) of $480 \pm 30 \mathrm{~km} \mathrm{~s}^{-1}$, where the error in the radial velocities arises primarily from the scatter in the line positions. We defer further discussion of the spectrum to the following section.

\subsubsection{Gemini/GMOS Spectroscopy}

2MASS J0616-6407 was also observed with the Gemini Multi-Object Spectrograph (Hook et al. 2004) mounted on the Gemini South Telescope on 2007 September 13 (UT). We used the 0.75 wide slit and $\mathrm{R} 400$ grating which yields a spectrum from 5900 to $10100 \AA$ with a resolving power of $R \sim 1300$. The OG515 filter was used to suppress stray light from shorter

\footnotetext{
8 IRAF is distributed by the National Optical Astronomy Observatories, which are operated by the Association of Universities for Research in Astronomy, Inc., under cooperative agreement with the National Science Foundation.
}

wavelengths. A total of five $1800 \mathrm{~s}$ exposures were acquired at two different central wavelengths and at two positions along the slit. Standard calibration frames including flat-field and $\mathrm{CuAr}$ lamp images as well as observations of the G2 V star HD 60402 for telluric correction were also obtained. The flux standard EG 21 (Hamuy et al. 1994) was observed on 2007 September 14 (UT) with the same instrument setup.

The data were first reduced using the IRAF Gemini GMOS package. The raw frames were first bias-subtracted (with a bias created using GBIAS) and flat-fielded (with a normalized flat field created with GSFLAT). The $\mathrm{OH}$ sky lines were then subtracted and then spectra were optimally extracted using the GSEXTRACT routine. Wavelength calibration was achieved using the CuAr lamp images. The spectra were then flux calibrated using the observations of EG 21 and the GSCALIBRATE routine. Telluric correction was achieved by linearly interpolating over regions of $\mathrm{O}_{2}$ and $\mathrm{H}_{2} \mathrm{O}$ absorption in the spectrum of HD 60402, dividing by the raw spectrum of HD 60402 , and then multiplying the results into the flux-calibrated spectrum of 2MASS J0616-6407.

Unfortunately, the resulting spectrum exhibits strong noise spikes at the wavelengths of nearly all the $\mathrm{OH}$ sky lines. Fringing in the detector makes the subtraction of the OH sky lines using low-order polynomials difficult. We therefore reextracted the raw spectrum of 2MASS J0616-6407 so that we could first pair subtract the images with 2MASS J0616-6407 at different positions along the slit. Although this did not completely eliminate the strong residuals, it did improve the spectrum dramatically. Finally we measured a radial velocity of $445 \pm 18 \mathrm{~km} \mathrm{~s}^{-1}$ using the same technique described in the previous section. The radial velocity measurements from the LDSS-3 and GMOS spectra agree within the errors and a weighted average of the two values gives $454 \pm 15 \mathrm{~km} \mathrm{~s}^{-1}$.

The LDSS-3 and GMOS spectra are shown in Figure 3. The GMOS spectrum has a $\mathrm{S} / \mathrm{N}$ of 10 to 20 while the LDSS- 3 has a lower S/N. The spectra exhibit band heads of $\mathrm{CaH}(7035 \AA)$, $\mathrm{TiO}(7053,8432 \AA), \mathrm{CrH}(8611 \AA), \mathrm{FeH}(8692,9896 \AA)$, and atomic lines of $\mathrm{K}$ I $(7665,7699 \AA), \mathrm{Rb}$ I $(7800,7948 \AA)$, Na I (8183, $8195 \AA$ ), and Cs I (8521 $)$ ). The heavily pressurebroadened $\mathrm{K}$ I doublet, strong $\mathrm{CrH}$ and $\mathrm{FeH}$ band heads, and $\mathrm{Cs} \mathrm{I}$ and $\mathrm{Rb}$ I lines are hallmark spectral features of $\mathrm{L}$ dwarfs (Kirkpatrick et al. 1999) while the presence of the $\mathrm{CaH}$ and $\mathrm{TiO}$ band heads are consistent with known late-type $\mathrm{M}$ and $\mathrm{L}$ subdwarf spectra (Burgasser et al. 2007). Overall the spectra agree well except longward of $\sim 9200 \AA$ where the LDSS-3 spectrum is systematically higher than the GMOS spectrum. Burgasser et al. (2007) found a similar offset between LDSS-3 and GMOS spectra of ultracool M subdwarfs and ascribed it to an unknown flux calibration error and/or the fact that the 9200 $\AA$ telluric $\mathrm{H}_{2} \mathrm{O}$ band was not corrected in their GMOS data. Our GMOS spectrum of 2MASS J0616-6407 has been corrected for telluric absorption so we can eliminate this as the cause of the mismatch. Since the cause remains unknown, we restrict further analysis to $\lambda<9200 \AA$.

\subsection{Near-Infrared Imaging}

An image of 2MASS J0616-6407 was taken on 2008 Jan 17 (UT) using the $1.5 \mathrm{~m}$ telescope at CTIO with the Caméra PAnoramique Proche InfraRouge (CPAPIR; Artigau et al. 2004) wide field-IR imager in the $J$ band. The object was observed close to the meridian at an airmass of 1.2 and with seeing conditions $\sim 1^{\prime \prime} .5$. We used a nine-point dither pattern with $10^{\prime \prime}$ offsets. Each exposure was $\sim 60 \mathrm{~s}$ amounting to a total integration time 


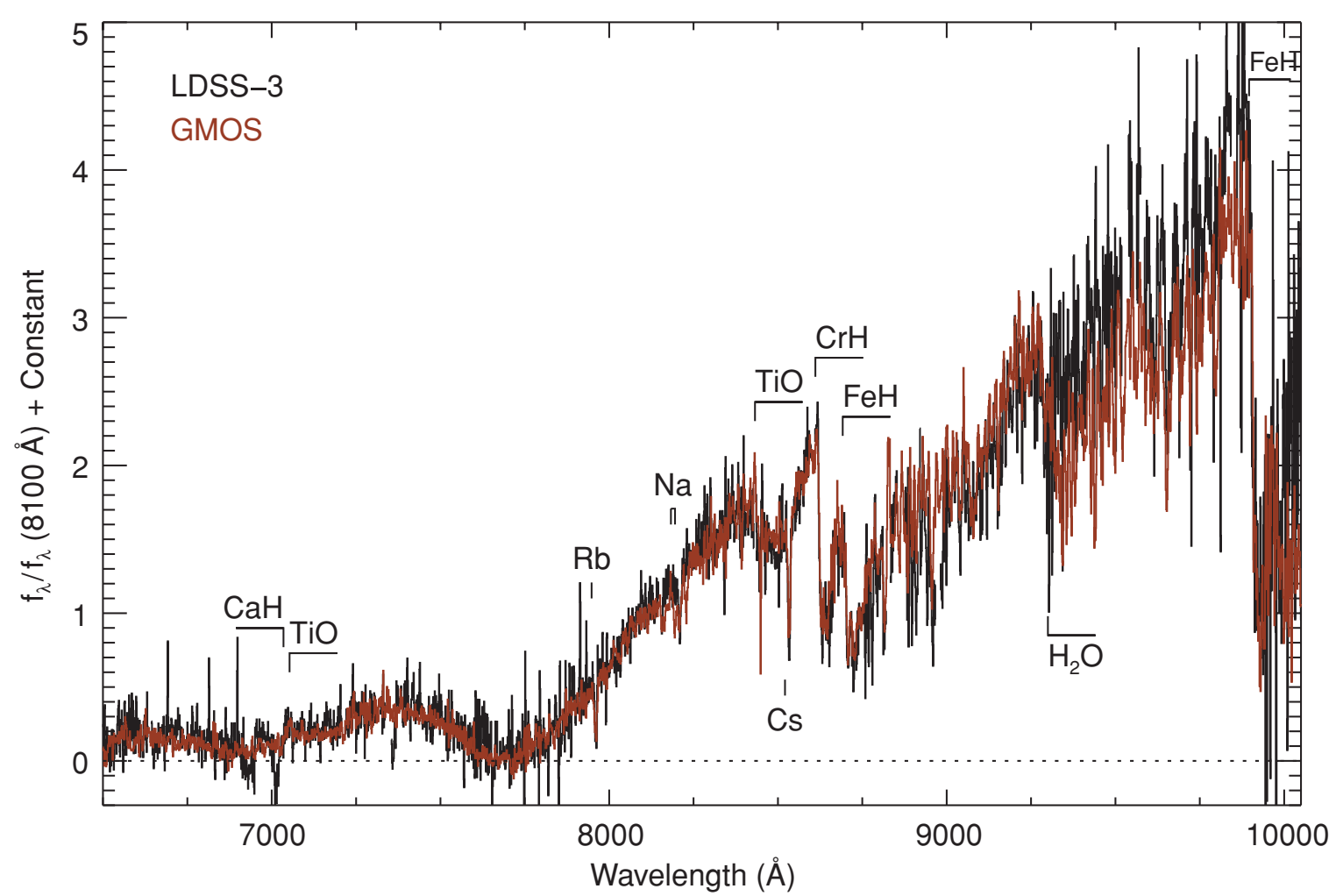

Figure 3. Red optical spectra of 2MASS J0616-6407 obtained with LDSS-3/Magellan (black) and GMOS/Gemini (red). The spectra are normalized to unity at $8100 \AA$. Prominent atomic and molecular absorption features are indicated.

(A color version of this figure is available in the online journal.)

of $\sim 9$ minutes. Science frames were first sky-subtracted using a sky frame created by median-combining all of the science data taken on a given night and then flat-fielded using a normalized dome flat. Individual frames were shifted and stacked to form the final combined image. The reduced science frame was astrometrically calibrated using the 2MASS Point Source catalog. We used a basic, six parameter, least-squares, linear transformation to obtain an astrometric solution for the CPAPIR image. The field-of-view of CPAPIR is $\sim 30^{\prime \prime}$ so there were over 1600 2MASS reference stars to calibrate CPAPIR to 2MASS. We limited our reference stars to the 450 stars with $12<J<15$ as objects in this intermediate magnitude range transformed with the smallest residuals from epoch to epoch. The solution reference stars were required to transform with total absolute residuals against 2MASS of $<0.2$ pixels (see Faherty et al. 2009 for more details). We measure a proper motion of $\mu_{\alpha}=+1404$

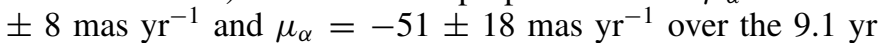
baseline between the CPAPIR image and the discovery 2MASS image. The errors were computed by combining the residuals of the astrometric solution, which accounted for 0.16 pixels, with the plate scale of CPAPIR $\left(\sim 1^{\prime \prime}\right.$ pixel $\left.^{-1}\right)$ under the given baseline. The positional uncertainty for 2MASS J0616-6407 was also calculated by comparing the residuals of transforming the $x, y$ position for our target over consecutive dithered images. However this uncertainty was negligible compared to the contribution from the astrometric solution.

\section{ANALYSIS}

\subsection{Spectral Classification}

Determining the spectral type of 2MASS J0616-6407 is not straightforward because an L subdwarf spectral classification scheme has yet to be rigorously defined in either the red optical or the near-infrared due to the few examples known. The depth of the $1.4 \mathrm{H}_{2} \mathrm{O}$ band and the shape of the $H$ band (see Figure 2) suggest that 2MASS J0616-6407 has a near-infrared spectral type intermediate between that of 2MASS J1626+3925 and 2MASS J0532+8246, perhaps sdL4 to sdL6. In order to derive a spectral type based on the red optical spectrum, we followed Burgasser et al. (2007) and compared the GMOS spectrum of 2MASS J0616-6407 to the spectra of field L dwarf spectral standards (Kirkpatrick et al. 1999). The best match is the L5 dwarf DENIS-P J1228.2-1547 resulting in an optical spectral type of sdL5 for 2MASS J0616-6407. Note that a similar comparison in the near-infrared is impossible because L dwarf spectral standards have yet to be defined at these wavelengths (see however J. Kirkpatrick et al. 2009, in preparation). Figure 4 shows the red optical spectra of both 2MASS J0616-6407 and DENIS -P J1228.2-1547. The strengths of the $\mathrm{CrH}$ and $\mathrm{FeH}$ band heads, width of the K I doublet, and overall $8000 \AA-9000 \AA$ slope of the two spectra agree well. Also shown in Figure 4 are the spectra of 2MASS J0532+8246 and 2MASS J1626+3925 along with their respective $\mathrm{L}$ dwarf standards. The strength of the features in the spectrum of 2MASS J0616-6407 are intermediate between that of 2MASS J1626+3925 and 2MASS $\mathrm{J} 0532+8246$, in good agreement with its derived spectral type of sdL5.

\subsection{Kinematics}

Although the high proper motion and large radial velocity of 2MASS J0616-6407 suggest that 2MASS J0616-6407 is a high-velocity star and thus possibly a member of the halo population, a distance is required in order to compute space velocities. We estimated a spectrophotometric distance to 


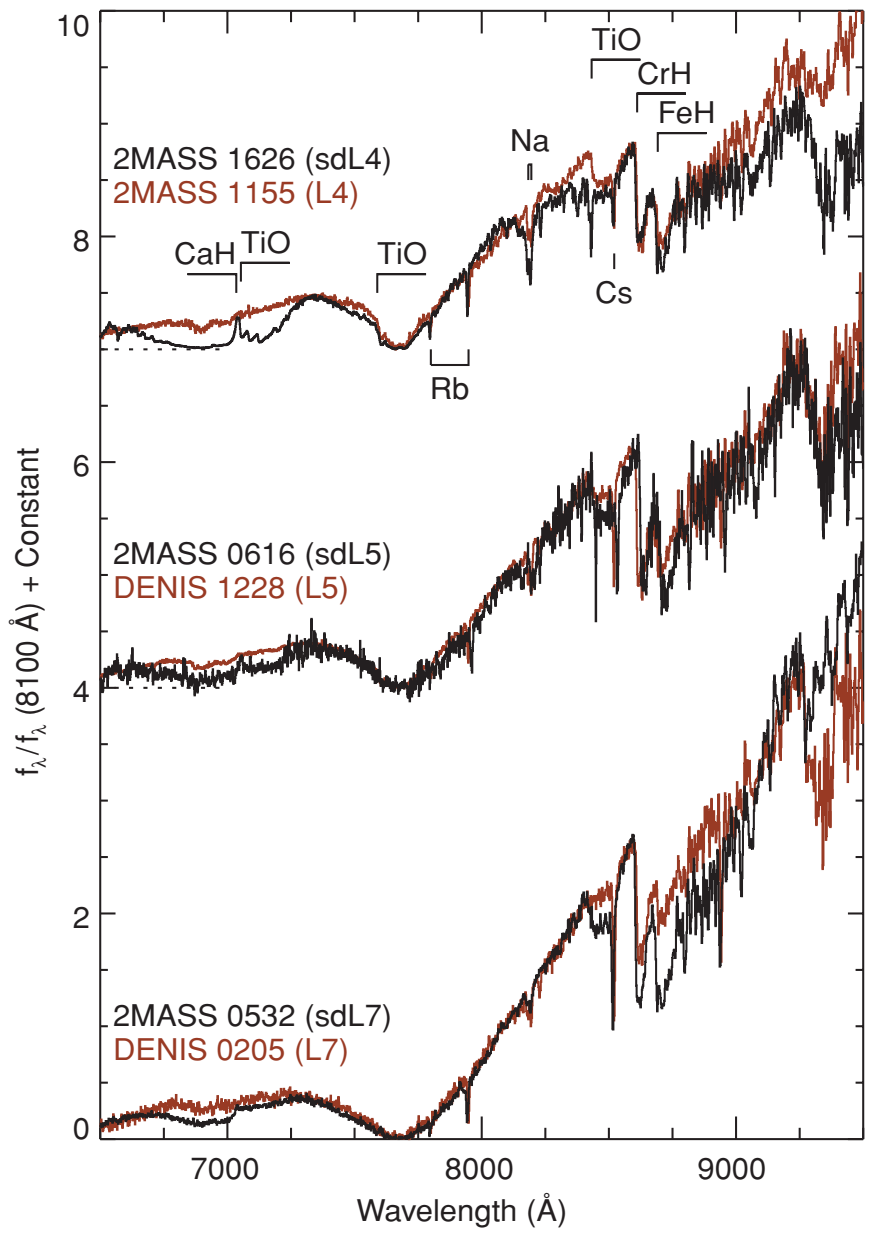

Figure 4. Red optical spectra of 2MASS J1626+3925 (sdL4; Burgasser et al. 2007), 2MASS J0616-6407, and 2MASS J0532+8246 (sdL7; Burgasser et al. 2003) compared to those of 2MASS 1155009+230706 (L4; Kirkpatrick et al. 1999), DENIS-P J1228.2-1547 (L5; Kirkpatrick et al. 1999), and DENISP J0205.4-1159 (L7; Kirkpatrick et al. 1999), respectively. The spectra are normalized to unity and offset by constants (dotted lines). Prominent atomic and molecular absorption features are indicated.

(A color version of this figure is available in the online journal.)

2MASS J0616-6407 in the following way. First we performed a weighted linear fit of the 2MASS $M_{J}, M_{H}$, and $M_{K s}$ values with respect to spectral type of the ultracool subdwarfs with known parallaxes (Monet et al. 1992; Dahn et al. 2008b; Schilbach et al. 2009; Burgasser et al. 2008), LHS 377 (sdM7), LSR 2036 + 5059 (sdM7.5), LSR J1425 + 7102 (sdM8), SSSPM 1013 - 1356 (sdM9.5), 2MASS J1626+3925 (sdL4) and 2MASS J0532+8246 (sdL7) and find,

$M_{J}=8.02+0.313 \times$ SpType,$\quad$ Cov $=\left(\begin{array}{cc}0.137 & -0.0114 \\ -0.0114 & 0.00106\end{array}\right)$

$M_{H}=7.77+0.300 \times$ SpType, $\quad \operatorname{Cov}=\left(\begin{array}{cc}0.148 & -0.0125 \\ -0.0125 & 0.00120\end{array}\right)$

$M_{K s}=7.44+0.320 \times$ SpType, $C o v=\left(\begin{array}{cc}0.172 & -0.0152 \\ -0.0152 & 0.00151\end{array}\right)$,

where SpType $=7$ for M7, SpType $=10$ for L0, etc., and Cov is the covariance matrix of the fit. For those dwarfs with two
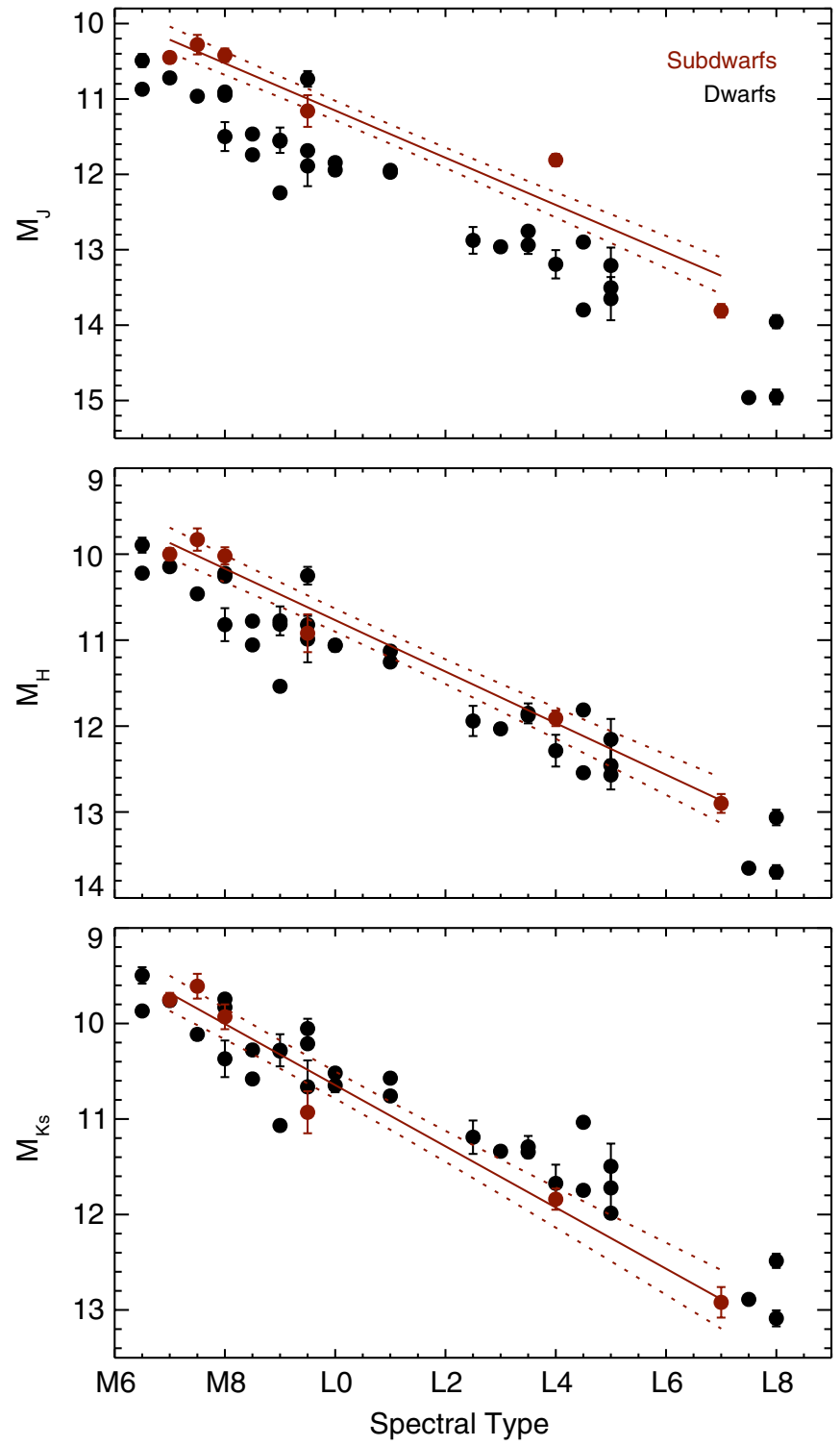

Figure 5. 2MASS $M_{J}, M_{H}$, and $M_{K s}$ values for LHS 377 (sdM7), LSR 2036+5059 (sdM7.5), LSR J1425+7102 (sdM8), SSSPM 1013-1356 (sdM9.5), 2MASS J1626+3925 (sdL4), and 2MASS J0532+8246 (sdL7) are shown in red. The linear fit to these values and the $\pm 1 \sigma$ range are shown as solid and dotted lines. Shown in black are a sample of field dwarfs from Dahn et al. (2002) and references therein that have not been identified as binaries in high-resolution imaging. All spectral types are based on optical spectroscopy.

(A color version of this figure is available in the online journal.)

parallax measurements, we used the weighted average of the two values to compute their absolute magnitudes. Figure 5 shows the absolute magnitudes of the subdwarfs along with those of single field $\mathrm{M}$ and L dwarfs from Dahn et al. (2002) and references therein. In comparison to the field dwarfs, the L subdwarfs appear overluminous in the $J$ band and underluminous in the $K$ band. If we assume that the effective temperature scale of the L dwarfs and subdwarfs are similar (see however Burgasser \& Kirkpatrick 2006; Burgasser et al. 2009), we can ascribe this behavior to a change in metallicity. At a fixed $T_{\text {eff }}$ and $g, \mathrm{~L}$ dwarfs become brighter in the $J$ band and fainter in the $K$ band with decreasing metallicity (Burrows et al. 2006) due primarily to the increasing importance of CIA $\mathrm{H}_{2}$ absorption in the $K$ band.

We estimate a distance of $57 \pm 9$ pc for 2MASS J0616-6407 using the absolute $J$ and $H$ magnitudes derived using Equations 

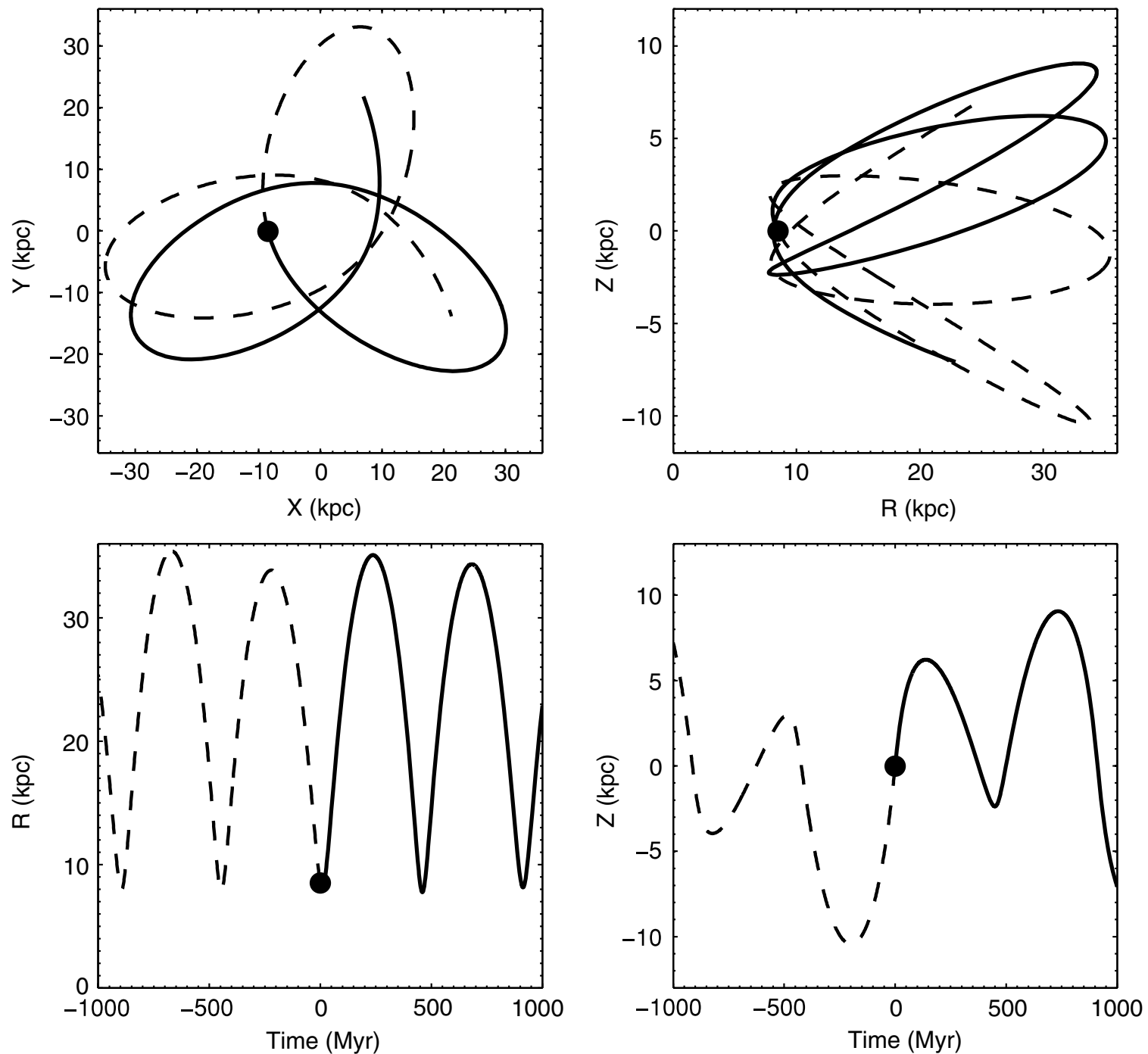



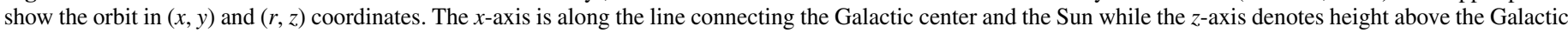


Sun is denoted with a solid circle.

(1) and (2) for a spectral type of sdL5 (2MASS J0616-6407 has only an upper limit in the $K_{s}$ band). The value is the weighted average of the two spectrophotometric distance estimates and the error includes the covariance terms and an error of \pm 0.5 a subtype in the spectral type. The estimated distance and proper motion of 2MASS J0616-6407 imply a tangential velocity $V_{\tan }$ of $379 \pm 60 \mathrm{~km} \mathrm{~s}^{-1}$ which is roughly an order of magnitude greater than the median value of field L dwarfs (Vrba et al. 2004; Faherty et al. 2009) and confirms that 2MASS J0616-6407 is a member of the halo population. Indeed, Faherty et al. (2009) found no M, L, and T dwarfs with $V_{\tan }>170 \mathrm{~km} \mathrm{~s}^{-1}$ in their sample of $634 \mathrm{~L}$ and $\mathrm{T}$ dwarfs and $456 \mathrm{M}$ dwarfs. We have also computed $(u, v, w)^{9}$ velocities of 2MASS J0616-6407 with respect to the Local Standard of Rest (LSR) following Johnson \& Soderblom (1987) updated with the J2000.0 galactic coordinate transformations of Murray (1989). Following the convention that the $u$ is positive toward the Galactic center $(l=0, b=0)$, we find $(u, v, w)=(94,-573,125) \pm(10,31,53) \mathrm{km} \mathrm{s}^{-1}$ after

\footnotetext{
9 We follow the convention that lower case letters refer to velocities with respect to the LSR while upper case letters refer to velocities with respect to the Sun (Mihalas \& Binney 1981, page 608).
}

correcting for a solar velocity with respect to the LSR of (10.00, $5.25,7.17) \mathrm{km} \mathrm{s}^{-1}$ (Dehnen \& Binney 1998). The LSR moves at $\Theta_{\mathrm{LSR}}=220 \mathrm{~km} \mathrm{~s}^{-1}(\Theta$ and $v$ point in the direction of Galactic rotation, $l=90, b=0$; Kerr \& Lynden-Bell 1986) in the rest frame of the Galaxy so 2MASS J0616-6407 has a highly retrograde orbit with $\Theta=-353 \mathrm{~km} \mathrm{~s}^{-1}$. The stars in the inner halo of the Galaxy have slightly prograde orbits with $\Theta=0$ to $50 \mathrm{~km} \mathrm{~s}^{-1}$ while the stars in the outer halo have retrograde orbits with $\Theta=-40$ to $-70 \mathrm{~km} \mathrm{~s}^{-1}$ (Carollo et al. 2007). The rotation of 2MASS J0616-6407 around the center of the Galaxy clearly indicates that 2MASS J0616-6407 is a member of the outer halo.

Based on its $(u, v, w)$ velocities we also computed the Galactic orbit of 2MASS J0616-6407 following Burgasser et al. (2009). We examined two simplified versions of the Galactic potential model described in Dehnen \& Binney (1998) composed of spherically-symmetric halo and bulge mass distributions and an axisymmetric, thin exponential disk. The two Galactic mass models were parameterized according to Binney \& Tremaine (2008, Table 2.3), which fit the measured rotation curve of the Galaxy but bracket the allowable ratios of disk to halo mass at the Solar radius. The orbit of 2MASS J0616-6407 was integrated 
using a second-order leapfrog method (kick-drift-kick) with a constant timestep of $1 \mathrm{kyr}$ over $\pm 1 \mathrm{Gyr}$ centered on the present epoch. Energy was conserved to better than 1 part in $10^{-4}$ over the full length of the simulation, with the error dominated by the resolution of the grid on which the disk force and potential were interpolated. The $z$-component of angular momentum was conserved to 1 part in $10^{-13}$.

Figure 6 shows the orbit of 2MASS J0616-6407 based on the halo-dominant mass model, plotted in the rest frame of the Galaxy. The $z$-coordinate is defined to be positive toward the Galactic center to align with our definition of $u$, and the Sun is located at $(-8.5,0,0.027) \mathrm{kpc}$ (Kerr \& Lynden-Bell 1986; Chen et al. 2001). Prograde motion in this reference frame is counterclockwise, so the orbit of 2MASS J0616-6407 is clearly retrograde, as well as wide and eccentric, with $R$ extending from 8 to $36 \mathrm{kpc}(e \sim 0.7$ from $\max / \min R$ ) over the simulation period. The orbit of 2MASS J0616-6407 also exhibits a fairly broad range of inclinations, with $z$ spanning $\pm 10 \mathrm{kpc}$ ( $\max |i| \sim 15$ degrees). For the disk-dominant mass model, the orbit is even wider (extending out to $40 \mathrm{kpc}$ ) but with similar eccentricity and inclination range. The breadth of this orbit and its retrograde motion support the conclusion that 2MASS J0616-6407 is a member of the outer halo, as this population dominates at $R>14-20 \mathrm{kpc}$ (Carollo et al. 2007). We note that our conclusions remain unchanged if 2MASS J0616-6407 is an equal magnitude binary.

\section{DISCUSSION}

The Galactic orbit of 2MASS J0616-6407 is distinctly different from that of other late-type subdwarfs (e.g., LSR J0822 + 1700, 2MASS J0532+8246, LSR J1425 + 7102, SDSS J1256-0224) which all have perigalacticons of $\lesssim 1 \mathrm{kpc}$ and apogalacticons near the Sun (Lépine et al. 2004; Burgasser et al. 2008, 2009; Dahn et al. 2008a; Burgasser et al. 2009). As noted by Burgasser et al. (2009), these other subdwarfs have kinematic properties which are consistent with membership in the inner halo of the Galaxy. 2MASS J0616-6407 is therefore the first late-type subdwarf that is a member of the outer halo and therefore may represent a new class of L subdwarfs. In particular, the inner halo comprises stars with a peak metallicity of $[\mathrm{Fe} / \mathrm{H}]$ $\sim-1.6$ while the outer halo has a peak near -2.2 (Carollo et al. 2007). This suggests that 2MASS J0616-6407 may be even more metal-poor than the other late-type subdwarfs known. However, the techniques needed to derive accurate metallicities of late-type subdwarfs are in their infancy (e.g., Burgasser et al. 2007, 2009) and consequently an accurate measurement of the metallicity of 2MASS J0616-6407 must await future work. Finally we note that the discovery of additional outer halo ultracool subdwarfs will be difficult given their intrinsic faintness and the large amount of time that they spend away from the solar neighborhood.

We thank Bill Vacca for fruitful discussions about the reduction of optical spectra and John Bochanski and Andrew West for clarifications on the Galactic orbit geometry. This publication makes use of data from the Two Micron All Sky Survey, which is a joint project of the University of Massachusetts and the Infrared Processing and Analysis Center, and funded by the National Aeronautics and Space Administration and the National Science Foundation, the SIMBAD database, operated at CDS, Strasbourg, France, NASA's Astrophysics Data System Bibliographic Services, the M, L, and T dwarf compendium housed at DwarfArchives.org and maintained by Chris Gelino,
Davy Kirkpatrick, and Adam Burgasser, and the NASA/ IPAC Infrared Science Archive, which is operated by the Jet Propulsion Laboratory, California Institute of Technology, under contract with the National Aeronautics and Space Administration. OSIRIS is a collaborative project between the Ohio State University and Cerro Tololo Inter-American Observatory (CTIO) and was developed through NSF grants AST 90-16112 and AST 92-18449. CTIO is part of the National Optical Astronomy Observatory (NOAO), based in La Serena, Chile. NOAO is operated by the Association of Universities for Research in Astronomy (AURA), Inc. under cooperative agreement with the National Science Foundation.

Facilities: Gemini (GMOS), SOAR (OSIRIS), LDSS-3 (Magellan), CTIO (CPAPIR).

\section{REFERENCES}

Artigau, E., Doyon, R., Vallee, P., Riopel, M., \& Nadeau, D. 2004, in SPIE Conf. Ser. 5492, ed. A. F. M. Moorwood \& M. Iye (Bellingham, WA: SPIE), 1479

Bessell, M. S. 1982, Proc. Astron. Soc. Aust., 4, 417

Binney, J., \& Tremaine, S. 2008, Galactic Dynamics (2nd ed.; Princeton, NJ: Princeton Univ. Press)

Borysow, A., Jorgensen, U. G., \& Zheng, C. 1997, A\&A, 324, 185

Burgasser, A. J. 2004, ApJ, 614, L73

Burgasser, A. J., Cruz, K. L., \& Kirkpatrick, J. D. 2007, ApJ, 657, 494

Burgasser, A. J., \& Kirkpatrick, J. D. 2006, ApJ, 645, 1485

Burgasser, A. J., Kirkpatrick, J. D., \& Lépine, S. 2005, in ESA Special Publication 560, 13th Cambridge Workshop on Cool Stars, Stellar Systems and the Sun, ed. F. Favata, G. A. J. Hussain, \& B. Battrick (Noordwijk: ESA), 237

Burgasser, A. J., Vrba, F. J., Lépine, S., Munn, J. A., Luginbuhl, C. B., Henden, A. A., Guetter, H. H., \& Canzian, B. C. 2008, ApJ, 672, 1159

Burgasser, A. J., Witte, S., Helling, C., \& Hauschildt, P. H. 2009, ApJ, in press Burgasser, A. J., et al. 2002, ApJ, 564, 421

Burgasser, A. J., et al. 2003, ApJ, 592, 1186

Burrows, A., Sudarsky, D., \& Hubeny, I. 2006, ApJ, 640, 1063

Carollo, D., et al. 2007, Nature, 450, 1020

Chen, B., et al. 2001, ApJ, 553, 184

Cushing, M. C., \& Vacca, W. D. 2006, AJ, 131, 1797

Cushing, M. C., Vacca, W. D., \& Rayner, J. T. 2004, PASP, 116, 362

Dahn, C. C., et al. 2002, AJ, 124, 1170

Dahn, C. C., et al. 2008a, ApJ, 686, 548

Dahn, C. C., et al. 2008b, ApJ, 686, 548

Dehnen, W., \& Binney, J. J. 1998, MNRAS, 298, 387

Epchtein, N., et al. 1997, The Messenger, 87, 27

Faherty, J. K., Burgasser, A. J., Cruz, K. L., Shara, M. M., Walter, F. M., \& Gelino, C. R. 2009, AJ, 137, 1

Geballe, T. R., et al. 2002, ApJ, 564, 466

Giclas, H. L., Burnham, R., \& Thomas, N. G. 1971, Lowell Proper Motion Survey Northern Hemisphere. The G Numbered Stars. 8991 Stars Fainter than Magnitude 8 with Motions $>0$ '.26/year (Flagstaff, AZ: Lowell Obs.)

Giclas, H. L., Burnham, R., Jr., \& Thomas, N. G. 1978, Lowell Obs. Bull., 8, 89 Gizis, J. E. 1997, AJ, 113, 806

Hamuy, M., Suntzeff, N. B., Heathcote, S. R., Walker, A. R., Gigoux, P., \& Phillips, M. M. 1994, PASP, 106, 566

Hook, I. M., Jørgensen, I., Allington-Smith, J. R., Davies, R. L., Metcalfe, N., Murowinski, R. G., \& Crampton, D. 2004, PASP, 116, 425

Johnson, D. R. H., \& Soderblom, D. R. 1987, AJ, 93, 864

Kerr, F. J., \& Lynden Bell, D. 1986, MNRAS, 221, 1023

Kirkpatrick, J. D. 2005, ARA\&A, 43, 195

Kirkpatrick, J. D., et al. 1999, ApJ, 519, 802

Leggett, S. K., Allard, F., Dahn, C., Hauschildt, P. H., Kerr, T. H., \& Rayner, J. 2000, ApJ, 535, 965

Leggett, S. K., Allard, F., Geballe, T. R., Hauschildt, P. H., \& Schweitzer, A. 2001, ApJ, 548, 908

Lépine, S., Rich, R. M., \& Shara, M. M. 2003, ApJ, 591, L49

Lépine, S., \& Scholz, R.-D. 2008, ApJ, 681, L33

Lépine, S., \& Shara, M. M. 2005, AJ, 129, 1483

Lépine, S., Shara, M. M., \& Rich, R. M. 2004, ApJ, 602, L125

Linsky, J. L. 1969, ApJ, 156, 989

Looper, D. L., Kirkpatrick, J. D., \& Burgasser, A. J. 2007, AJ, 134, 1162

Luyten, W. J. 1979, LHS Catalogue. A Catalogue of Stars with Proper Motions Exceeding 0"5 Annually (2nd ed., Minneapolis, MN: Univ. Minnesota) 
Mihalas, D., \& Binney, J. 1981, Galactic Astronomy: Structure and Kinematics (2nd ed.; San Francisco, CA: W. H. Freeman)

Monet, D. G., Dahn, C. C., Vrba, F. J., Harris, H. C., Pier, J. R., Luginbuhl, C. B., \& Ables, H. D. 1992, AJ, 103, 638

Murray, C. A. 1989 , A\&A, 218, 325

Pokorny, R. S., Jones, H. R. A., Hambly, N. C., \& Pinfield, D. J. 2004, A\&A, 421, 763

Ralchenko, Y., Kramida, A. E., \& Reader, J. NIST ASD Team 2008, NIST Atomic Spectra Database (version 3.1.5), Tech. Rep. (Gaithersburg, MD: National Institute of Standards and Technology), http://physics.nist.gov/asd3

Reid, I. N., Burgasser, A. J., Cruz, K. L., Kirkpatrick, J. D., \& Gizis, J. E. 2001, AJ, 121, 1710
Reiners, A., \& Basri, G. 2006, AJ, 131, 1806

Saumon, D., Bergeron, P., Lunine, J. I., Hubbard, W. B., \& Burrows, A. 1994, ApJ, 424, 333

Schilbach, E., Roeser, S., \& Scholz, R. 2009, A\&A, 493, L27

Sivarani, T., Lépine, S., Kembhavi, A. K., \& Gupchup, J. 2009, ApJ, 694, L140 Skrutskie, M. F., et al. 2006, AJ, 131, 1163

Tody, D. 1986, in SPIE Conf. Ser. 627, Society of Photo-Optical Instrumentation Engineers (SPIE) Conference Series, ed. D. L. Crawford (Bellingham, WA: SPIE), 733

Vacca, W. D., Cushing, M. C., \& Rayner, J. T. 2003, PASP, 115, 389

Vrba, F. J., et al. 2004, AJ, 127, 2948

York, D. G., et al. 2000, AJ, 120, 1579 